\title{
Estudo sobre dimensôes da avaliação da Estratégia Saúde da Família pela perspectiva do usuário
}

I ${ }^{1}$ Verena Duarte Moraes, ${ }^{2}$ Carlos Eduardo Aguilera Campos,

${ }^{3}$ Ana Laura Brandão I

Resumo: A satisfação dos usuários é um dos componentes básicos da qualidade de qualquer serviço de saúde. Tem sido crescente a incorporação desse aspecto em estudos avaliativos de unidades e equipes de Atenção Básica à Saúde. No Brasil, esse processo ainda é incipiente, com poucos instrumentos disponíveis, adequados e validados. O objetivo deste trabalho foi investigar as dimensões mais valorizadas pelos usuários. Sua metodologia contou com a realização de dois grupos focais em duas unidades básicas de saúde (UBS) com contextos distintos para ampliar as possibilidades de resposta. Foram feitas análise de conteúdo e categorização das falas em dimensōes e subdimensões da satisfação. Chegou-se a um total de 17 dimensões, sendo as mais citadas: 1) acesso e disponibilidade do serviço de saúde; 2) organização dos processos de trabalho; 3) relação com os profissionais de saúde; 4) longitudinalidade do cuidado e estabelecimento de vínculo entre profissionais e usuários; e 5) coordenação do cuidado. Conclui-se que conhecer as dimensões da satisfação mais valorizadas pelo usuário é essencial para a construção de instrumentos mais adequados ao contexto da ESF no país.

1 Residente de Saúde Coletiva do IESC-UFRJ. Rio de Janeiro, Brasil. Endereço eletrônico: moraes.veve@gmail.com

2 Professor Adjunto Faculdade de Medicina-UFRJ. Rio de Janeiro, Brasil. Endereço eletrônico: cadu@vetor.com.br

${ }^{3}$ Pesquisadora Teias Manguinhos, ENSP-Fiocruz. Rio de Janeiro, Brasil. Endereço eletrônico: alaurabrandao@ gmail.com

Recebido em: 05/02/2013 Aprovado em: 13/01/2014 


\section{Introdução}

No Brasil, os estudos de satisfação destacam-se a partir da segunda metade da década de 1990, devido à maior da participação da comunidade nos processos de avaliação e planejamento, assim como à promoção da noção de accountability (prestação de contas) (AHARONY; STRASSER, 1993; SITZIA; WOOD, 1997). É provável que o desenvolvimento do conceito de satisfação tenha reforçado o movimento do consumidor e suas demandas. Esse conceito, assim como o usuário, passou a ser visto como uma figura central do accountability em todos os serviços públicos. Ao longo dos últimos dez anos, a satisfação do consumidor ganhou reconhecimento difuso como uma medida de qualidade em muitos serviços do setor público (WILLIANS, 1994).

Considerando a satisfação do cliente, cita-se a European Foundation for Quality Management (EFQM), que desenvolveu em 1991 o modelo de excelência da EFQM, baseado no conceito da gestão pela qualidade total. Um dos princípios fundamentais da EFQM é o foco no cliente. Tal conceito preconiza que a organização deve estar orientada para a satisfação das necessidades expressas e potenciais dos clientes externos e internos da organização. Devem ser identificados, tanto quanto possível, as necessidades dos clientes e os esforços empreendidos no sentido de conhecê-los o melhor possível (ANDRADE, 2004).

No que tange aos serviços de saúde, o interesse pela temática tem sido reforçado. Estudos mostraram que usuários satisfeitos têm melhor adesão às recomendações e planos terapêuticos, o que contribui para sua saúde física e mental e melhora também sua qualidade de vida, além de favorecer a continuidade do tratamento (BARON-EPEL, 2001; GULDVOG, 1999; ESPERIDIÃO; TRAD, 2006). Essa relação com a satisfação e a efetividade do cuidado parece ser uma ideia-chave para convencer os gestores e profissionais sobre a importância desses estudos.

Há que se sinalizar, porém, que o termo "satisfação", muito utilizado nas sondagens aos usuários de serviços de saúde, é um conceito vago que se refere a realidades variadas (ESPERIDIÃO; TRAD, 2006). Destaca-se que não existe um consenso sobre o conceito de satisfação; entretanto, a forma mais frequente para determinar esse conceito é em termos das expectativas e da percepção dos usuários em relação aos serviços recebidos. Assim, o que se mede, muitas vezes, não é exatamente a satisfação, mas a percepção e a expectativa prévia dos usuários (PARASURAMAN, 1988). A avaliação por parte dos usuários é difícil de ser medida, pois trata-se de mensuraçôes de opiniões influenciadas por variadas 
expectativas, anteriores e atuais (KOTAKA, 1997). Entre os vários determinantes

prováveis da satisfação do paciente com os cuidados de saúde estão suas atitudes e percepçôes antes de experimentar o cuidado (LINDER-PELZ, 1982).

Existem diversos fatores que influenciam a satisfação dos usuários dos serviços de saúde. A interação entre eles resulta em um determinado nível de aceitabilidade e satisfação. Trata-se de uma interação complexa, já que tratamos de um aspecto de base subjetiva, baseado em percepções e experiências (SECLÉN-PALACIN; DARRAS, 2000).

As múltiplas dimensões envolvidas com a satisfação devem ser conhecidas por serem essenciais para o desenvolvimento de instrumentos confiáveis. Os instrumentos comumente utilizados na avaliação da satisfação pela perspectiva do usuário são, em sua maioria, questionários de opiniões com alternativas fechadas para questôes previamente formuladas, com escala do tipo Likert. Assim, para os serviços avaliarem significativamente as experiências e percepções dos usuários, deve-se primeiro realizar uma pesquisa para identificar e entender os mecanismos através dos quais esses pacientes percebem e avaliam os serviços (WILLIANS, 1994).

Os indivíduos devem ser reconhecidos como sujeitos capazes de avaliar e intervir, modificando o próprio sistema, fortalecendo, assim, o fazer democrático da saúde. É uma oportunidade de se verificar, na prática, a resposta da comunidade à oferta do serviço de saúde, como também a melhor adequação do serviço às expectativas da sua comunidade (TEIXEIRA, 2004). Parte-se aqui das premissas de que os pacientes (e também os cuidadores) são bons juízes da qualidade dos cuidados de saúde que recebem e de que existem boas técnicas para se apurar o ponto de vista dos usuários (RIGGE, 2009). Este estudo teve como objetivo identificar as dimensões da satisfação mais valorizadas pelos usuários.

\section{Métodos}

Optou-se por utilizar a técnica de grupo focal. Essa técnica produz informações mais rápidas e com menor custo quando comparada às entrevistas individuais, além de ser flexível, permitindo sondar atitudes e opiniōes que poderiam não ser reveladas em um questionário. Como o grupo focal tem o propósito de obter informações de caráter qualitativo em profundidade, fornece aos gerentes de projetos ou instituições uma grande riqueza de informações qualitativas sobre o desempenho de atividades desenvolvidas, prestação de serviços, novos produtos e outras questões (GOMES; BARBOSA, 1999). Além disso, é uma técnica bem 
aceita nas comunidades, pois o grupo de discussão é uma forma de comunicação muito comum nesses contextos (DAWSON et al., 1993).

Foram realizados dois grupos focais em unidades de saúde distintas. O primeiro foi realizado em um Centro de Saúde Escola (CSE) que contava com sete equipes de saúde da família. O segundo, em um Centro Municipal de Saúde (CMS), que, além dos profissionais especialistas pertencentes ao modelo tradicional de atenção à saúde, possuía também quatro equipes de saúde da família. Esse tipo de CMS é denominado "unidade mista".

Os grupos contaram com a presença de oito usuários. Segundo Krueger e Casey (1996), cada sessão de grupo focal deve ser composta por no mínimo quatro e no máximo doze usuários, visto que deve garantir a oportunidade dos membros de expor suas opinióes e, por outro lado, garantir a diversidade de ideias. Foram critérios de elegibilidade: ser maior de 18 anos e ter cursado no mínimo a $3^{\circ}$ série de escolaridade. Os grupos foram compostos por usuários de ambos os sexos, com faixa etária diversificada.

Foi utilizado um roteiro para auxiliar o mediador no desenvolvimento do grupo focal e um questionário para a obtenção de informações dos usuários contendo: faixa etária, sexo, nível de escolaridade, autopercepção de saúde. Em relação esta, havia cinco possibilidades de resposta: ótima, muito boa, boa, regular e má. A análise do perfil dos usuários é importante, uma vez que ele pode estar interligado ao conceito de satisfação, interferindo nas dimensões citadas.

Foi realizada análise de conteúdo por procedimentos sistemáticos e objetivos de descrição do conteúdo das mensagens. Buscou-se levantar indicadores (quantitativos ou não) que permitam a inferência de conhecimentos relativos às mensagens (BARDIN, 1979). O estudo foi aprovado pelo Comitê de Ética do IESC/UFRJ (Processo N o 56/2011).

\section{Resultados}

O primeiro grupo realizado teve a participação de três usuários do sexo feminino e um do sexo masculino. Já a segunda sessão do grupo focal foi composta por dois usuários de sexo masculino e dois do feminino. A faixa etária dos participantes variou de 30 a 69 anos. Os usuários pertencentes ao CSE tinham menor escolaridade quando comparados ao outro grupo.

Em relação à autopercepção da saúde, o primeiro grupo considerou seu estado de saúde melhor, com as respostas "ótima", "muito boa", "boa" e "regular", quando comparado ao segundo grupo, que só obteve respostas "má" e "regular" (quadro 1). 


\begin{tabular}{|c|c|c|c|}
\hline & Grupo focal 1 & Grupo focal 2 & Total \\
\hline Características dos usuários & $\mathbf{n}$ & $\mathbf{n}$ & \\
\hline \multicolumn{4}{|l|}{ Sexo } \\
\hline Feminino & 3 & 2 & 5 \\
\hline Masculino & 1 & 2 & 3 \\
\hline \multicolumn{4}{|l|}{ Idade } \\
\hline \multicolumn{4}{|l|}{$18-29$} \\
\hline $30-39$ & 2 & & 2 \\
\hline $40-49$ & 2 & & 2 \\
\hline $50-59$ & & 3 & 3 \\
\hline $60-69$ & & 1 & 1 \\
\hline \multicolumn{4}{|l|}{$70-79$} \\
\hline \multicolumn{4}{|l|}{$>80$ anos } \\
\hline \multicolumn{4}{|l|}{ Escolaridade } \\
\hline \multicolumn{4}{|l|}{ Analfabeto } \\
\hline Ensino Elementar incompleto & 2 & & 2 \\
\hline \multicolumn{4}{|l|}{ Fundamental incompleto } \\
\hline $\begin{array}{l}\text { Ensino Fundamental completo ou } \\
\text { Médio incompleto }\end{array}$ & 1 & 1 & 2 \\
\hline \multicolumn{4}{|l|}{ Superior incompleto } \\
\hline \multicolumn{4}{|l|}{ Superior Completo } \\
\hline \multicolumn{4}{|l|}{ Autopercepção de saúde } \\
\hline Ótima & 1 & & 1 \\
\hline Muito Boa & 1 & & 1 \\
\hline Boa & 1 & 1 & 2 \\
\hline Regular & 1 & 2 & 3 \\
\hline Má & & 1 & 1 \\
\hline
\end{tabular}

Fonte: elaboração própria (2013). 
Foram encontradas 17 dimensões e subdimensões de satisfação mais valorizadas pelos usuários. A frequência com que tais dimensões apareceram nas falas dos usuários variou muito. As mais citadas foram: acesso/disponibilidade, organização, relação profissional usuário, longitudinalidade e coordenação do cuidado (quadro 2).

Quadro 2. Dimensōes e subdimensões da satisfação observadas nos grupos focais. Rio de Janeiro, Brasil

\begin{tabular}{|c|c|c|c|c|}
\hline Dimensões & Subdimensões & $\begin{array}{l}\text { Grupo } \\
\text { focal } 1\end{array}$ & $\begin{array}{c}\text { Grupo } \\
\text { Focal } 2\end{array}$ & Total \\
\hline \multirow[t]{3}{*}{ Acesso } & & 16 & 31 & 47 \\
\hline & Burocracia & & & \\
\hline & Transporte & & & \\
\hline \multirow[t]{7}{*}{ Organizacional } & & 19 & 10 & 29 \\
\hline & Visita domiciliar & & & \\
\hline & Marcação de consulta & & & \\
\hline & Tempo de espera & & & \\
\hline & Duração da consulta & & & \\
\hline & Horário de funcionamento & & & \\
\hline & Rede de serviços em saúde & & & \\
\hline \multirow[t]{4}{*}{ Relacional } & & 11 & 10 & 21 \\
\hline & Característica do profissional & & & \\
\hline & Conduta do médico & & & \\
\hline & Vínculo & & & \\
\hline \multirow[t]{3}{*}{ Longitudinalidade } & & 4 & 8 & 12 \\
\hline & Vínculo & & & \\
\hline & Cuidados médicos & & & \\
\hline $\begin{array}{l}\text { Coordenação do } \\
\text { cuidado }\end{array}$ & & 1 & 10 & 11 \\
\hline & Referência/continuidade & & & \\
\hline
\end{tabular}




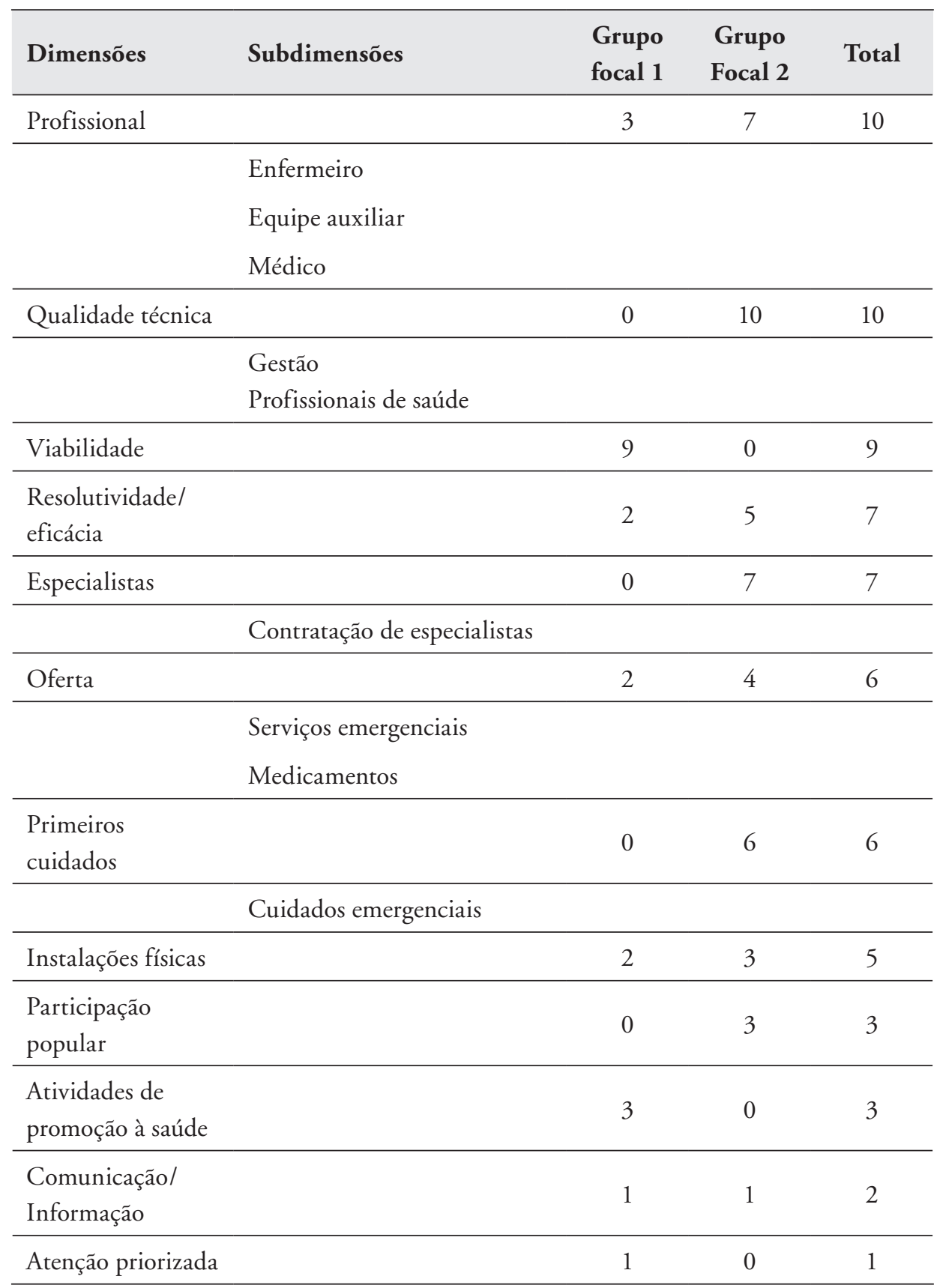

Fonte: elaboração própria (2013). 


\section{Discussão}

O perfil encontrado no estudo quanto ao gênero é semelhante ao descrito por outros estudos (BRANDÃO, 2011; COTTA et al., 2005; JORGE et al., 2007; MISHIMA et al., 2004; RONZANI; SILVA, 2008; SILVA; RIBEIRO; SILVEIRA, 2004; TEIXEIRA, 2004). A mulher é referência familiar sobre questôes de saúde, pois é ela quem avalia e toma a decisão de buscar atendimento. Desse modo, ela tem modos de perceber, classificar e agir sobre questóes relativas à saúde e doença (QUEIROZ, 1993). Para Trad et al. (2002), a perspectiva das mulheres como avaliadoras de serviços de saúde é caracterizada pelo profundo conhecimento sobre esse cotidiano.

Quanto à escolaridade, dois usuários tinham ensino médio completo e o restante apresentava grau de escolaridade inferior. Esse perfil encontra-se de acordo com outros estudos (COTTA et al., 2005; JORGE et al., 2007; HALAL et al., 1994; MISHIMA et al., 2004; SILVA; RIBEIRO; SILVEIRA, 2004; TEIXEIRA, 2004). Trad et al. (2002) afirmam que o Programa de Saúde da Família (PSF) seguiu uma tendência de se concentrar nas populaçōes mais pobres e com piores condiçôes de infraestrutura básica, o que implica uma baixa escolaridade. A faixa etária foi elevada, composta por indivíduos entre 30 a 69 anos.

Segundo Szwarcwald et al. (2005), a percepção da própria saúde é considerada um bom marcador do estado de saúde da população. Os resultados obtidos no presente estudo, para o estado de saúde autorreferido, mostraram que a maioria dos usuários indica ter uma saúde regular, o que está de acordo com o estudo de Brandão (2011), em que 40\% dos usuários referiram-se a esse nível de saúde.

Esse resultado pode estar relacionado ao perfil etário e à prevalência do sexo feminino, já que, à medida que a idade aumenta, o estado de saúde percebido tende a piorar, e as mulheres tendem a avaliar seu estado de saúde pior em relação aos homens. Também há relação com a renda baixa, que tem pior avaliação do estado de saúde quando comparada a grupos de maior renda (BARATA, 2008).

As cinco dimensões mais valorizadas pelos usuários serão abordadas mais detalhadamente. A questão do acesso foi a mais citada, revelando sua importância para os usuários. Ela denota que a população ainda tem muitas dificuldades para acessar o sistema de saúde, em especial a consulta médica.

Seria ótimo se eu chegasse aqui de manhã e recebesse meu atendimento, trazer minha neta e ela tivesse atendimento, não chegar aqui o profissional dizer para mim 'a senhora volta amanhã’ [...] (G2-Int1). 
Por ser a porta de entrada para os usuários, o acesso torna-se uma questão crucial para a Estratégia saúde da Família (ESF). Para Campbell, Roland e Buetow (2000) o acesso e a efetividade são as duas principais dimensões para a qualidade do cuidado. Segundo Starfield (2002), a acessibilidade é o elemento "estrutural". Sem acesso, a atenção será postergada, podendo afetar adversamente o diagnóstico e o manejo do problema.

Para os usuários, o acesso está vinculado à obtenção do atendimento médico (Stralen et al., 2008). Ainda que não devamos reduzir a atenção primária à prestação de consultas médicas, esse aspecto não pode ser minimizado. Apesar da dificuldade da população na obtenção de consultas, percebe-se que a expansão da ESF e sua localização mais próxima da população impactam positivamente.

Depois da construção da clínica de família com atendimento em casa para mim, para minha família, para meus vizinhos, eu vejo que melhorou muito. Antigamente não era assim (G1-Int1).

Por outro lado, percebe-se que a equipe de saúde da família não supre as necessidades da realização de exames e assistência especializada, com barreiras geográficas e burocráticas relativas à obtenção desses exames e dessas consultas.

Quando necessitam de exames não oferecidos pela Atenção Básica (AB), os usuários referem que são deslocados para lugares muito distantes de sua moradia ou têm de aguardar para obtê-los.

Eles mandam a gente fazer uma tomografia lá na Ilha do Governador. A gente vai sair daqui sem fisioterapia, de Santa Cruz, pra ir lá pra Ilha... Às vezes tem que sair daqui quatro e meia da manhã pra ir fazer uma fisioterapia lá (G2-Int1).

Além das barreiras geográficas, o excesso de etapas administrativas também dificulta a obtenção da consulta com os especialistas e de exames mais complexos. Os usuários relatam que no modelo antigo podiam procurar atendimento nos níveis secundários de assistência, e que agora necessitam passar primeiro pelo generalista.

[...] fui pra clínica, pedi ao médico pra me dar o encaminhamento; ele falou que o agente levaria a marcação pra mim com a minha receita. Eu estou aguardando até hoje [...] Então, quando eu andava com minhas pernas, eu ia lá procurar, eu ia para buscar, eu conseguia. Agora fiquei amarrada [...] (G2-Int2).

Ressalta-se que essa dificuldade está presente no sistema como um todo e que a ESF não pode isoladamente desburocratizar a assistência. É necessário um planejamento conjunto envolvendo os três níveis de assistência na perspectiva de fortalecer a atuação em rede de atenção à saúde. 
Transporte e burocracia foram as duas subdimensōes do acesso citadas. A proximidade dos serviços de saúde com o local de moradia dos usuários é um importante componente do acesso. Além da barreira geográfica, devem-se eliminar barreiras administrativas, e a unidade de $\mathrm{AB}$ deve organizar o fluxo na busca coordenada pela ESF, ofertando ao usuário a melhor opção de assistência disponível para cada tipo de necessidade.

A dimensão organizacional apareceu em segundo lugar, sendo mencionada em grande parte de forma negativa. A falta de organização interna dos serviços de saúde foi percebida pelos usuários em diversos aspectos. Nesta pesquisa foram citadas várias subdimensões, tais como: visita domiciliar, marcação de consulta, tempo de espera, duração da consulta, horário de funcionamento, rede de serviços em saúde.

Não cadastraram minha família [...] no início estava aquele negócio que iam de casa em casa. Até agora não chegaram na minha casa [...] (G2-Int4).

$\mathrm{Na}$ minha casa só foi quando inaugurou (G2Int2).

Tem uma lista, tipo equipe 3, ela tem uma lista que pertence a ela - 'vamos ver qual foi a família específica que a gente não atendeu, quais foram as ruas que a gente ainda não entrou' [...] (G2-Int1).

Estas falas podem refletir a falta de organização do processo de trabalho das equipes. Um dos fundamentos da $\mathrm{AB}$ é a adscrição dos usuários, conceituada como um processo de vinculação às equipes, que visa estabelecer a referência para o seu cuidado e a responsabilização entre as equipes.

Não é só para idoso. Se você tiver um problema, operou alguma coisa, eles também vão. Não é só para idoso. Vai aquelas menininhas, elas sempre vão à sua casa saber: e aí, tá tudo bem? (G1-Int1).

A subdimensão "marcação de consulta" foi descrita de forma negativa pelos usuários. Isso reflete problemas de organização das agendas dos profissionais, o que pode desmotivar os usuários e gerar dificuldade de acesso. Santos et al. (2007) afirmam que é necessário readequar os horários de marcação de consultas aos ritmos do dia a dia das populações abrangidas para proporcionar um acesso mais facilitado aos cuidados.

Aí, quando vem na data, tem que remarcar de novo, porque teve um feriado, uma paralisação aqui na fundação, teve greve [...] (G1-Int1).

A falta de organização interna no processo de trabalho das equipes é um fator que prejudica outros aspectos do cuidado, assim como interfere no estabelecimento 
de algumas diretrizes da ESF. A desorganização nas visitas domiciliares pode ocasionar a dificuldade para a marcação de consultas. A marcação de consultas sem planejamento, com números excessivos de usuários para o mesmo dia ou usuários no mesmo horário, possivelmente interferirá no tempo de espera do usuário, assim como no tempo que o profissional de saúde dispensa para cada consulta.

Percebem-se as subdimensões "tempo de espera" e "duração da consulta" nas falas abaixo:

Porque tem uns que ficam meia hora com a médica. Eu fiquei 10 minutos. Já era 17h30, ia dar 18h00, aí ela me atendeu rápido [...] (G1-Int2).

Isso influencia sim, porque aí o outro vai ficar esperando muito mais tempo, né? (G1-Int1).

Deve-se considerar, porém, que a população ainda tem uma prática antiga de acordar de madrugada e enfrentar fila para conseguir um atendimento. Dessa forma, as pessoas, mesmo com consultas programadas, chegam cedo à unidade, o que implica maior tempo de espera (SANTOS et al., 2007). A insatisfação com o horário de funcionamento fica clara na fala abaixo:

Vai almoçar é 13:30, 14:00, 15:00 horas. Não tem como, vem correndo porque tem um monte cliente lá. Não pode (G1-Int1).

Os usuários percebem que os problemas encontrados com a marcação de consultas, o tempo de espera, a duração da consulta e o horário de funcionamento podem estar relacionados à demanda excessiva. Entretanto, isso não pode justificar completamente tais problemas, já que os serviços devem ser organizados conforme as necessidades da população e projetados de forma que existam profissionais e unidades suficientes em cada nível de assistência. Um grande problema ainda se encontra na coordenação dos três níveis de sistema de saúde: a atenção primária, secundária e terciária.

Superlotação, como a maioria dos outros hospitais estão. Super lotação. (G1-Int1).

A falta de padronização do que deve ou não ser atendido pelo especialista causa um fluxo desorganizado, prejudicando todos os níveis de assistência. A organização interna dos serviços é primordial para que o trabalho em equipe seja eficaz e para que sejam ofertados cuidados de qualidade para os usuários, proporcionado vantagens para esses dois atores em relação aos papéis, direitos e deveres de todos. 
A dimensão relacional, muito citada na literatura, foi a terceira dimensão mais mencionada, sendo encontradas as subdimensões: "característica do profissional", "conduta do médico" e "vínculo". Tal dimensão é composta por muitos atributos, como: a forma como o médico conduz sua consulta, suas habilidades de comunicação, acolhimento, escuta, entre outras. Prévost, Fafard e Nadeau (1998) caracterizam a dimensão relacional como respeito e consideração, escuta, compreensão, respeito às decisões, acolhida, gentileza, respeito à privacidade, confidencialidade e discrição. Foram obtidos relatos positivos e negativos sobre a interação médico-paciente.

[...] porque aqui a gente vê muito isso, né? A maioria dos médicos são super dedicados. (G1-Int1)

[...] a abordagem, o interpessoal dela é ruim [...]. (G2-Int4)

A fala abaixo representa a subdimensão relacionada às características do profissional, isto é, gentileza, simpatia, personalidade e acolhimento ao usuário:

Os agentes não são capacitados para receber a pessoa, que é uma coisa simples, só te atender bem. É uma questão humana. Eles não estão sendo humanos. Eu vejo atendendo uma pessoa idosa - que nem agora: a senhora tá ali, com pressão alta, tá sentada ali na cadeira, não seria melhor ela estar deitada lá dentro [...]. (G2-Int1)

Destaca-se que houve falas positivas sobre os médicos especialistas que não pertencem às equipes de saúde da família.

Para mim falar de valorização [sic] eu preciso falar dos funcionários antigos [...]. (G2-Int4).

No presente estudo, o vínculo, como citado anteriormente, também foi considerado uma subdivisão da dimensão relacional. Segundo Mishima et al. (2010), a interação entre o usuário e a equipe se conforma na expectativa de "bons encontros" que os usuários esperam ter na unidade de saúde. A outra subdimensão, "conduta do médico", caracteriza-se pela forma como o este conduz sua consulta, com destaque para a qualidade clínica que desenvolve durante o atendimento, o que pode ser observado na menção:

Foi o que eu falei: a médica, ela é atenciosa, ela pergunta, examina direitinho. (G1-Int2)

Os usuários criticaram a conduta de médicos de unidades de pronto atendimento (UPA):

Às vezes você chega no médico ele nem encosta em você. É rapidinho: passa receita, é febre, é virose e pronto, acabou (G1-Int2). 
[...] examina, bota a mão, tira a pressão, vê com a coisinha pra respirar. (G1-Int3).

As interaçôes entre profissionais e pacientes contribuem para o estabelecimento de relaçôes de longa duração, que facilitam a efetividade na ESF. Apesar das interaçôes entre pacientes e profissionais ocorrerem no decorrer da consulta e do encaminhamento, são a amplitude e a profundidade do contexto que distinguem as interações na $\mathrm{AB}$ daquelas de outros níveis de atenção (STARFIELD, 2002).

A longitudinalidade do cuidado (quarta dimensão encontrada) pressupõe a continuidade da relação clínica, com estabelecimento de vínculo e responsabilização entre profissionais e usuários ao longo do tempo e de modo contínuo, acompanhando os efeitos e as consequências das intervenções em saúde, aperfeiçoando as condutas quando necessário, evitando a perda de referências e minimizando os riscos de iatrogenia decorrentes do desconhecimento das histórias de vida e da coordenação do cuidado. (BRASIL, 2011).

Para Starfield (2002), "continuidade da atenção" é uma expressão usada frequentemente para descrever a quantidade de vezes que os pacientes vão às unidades de saúde ou consultam o mesmo profissional, entre uma consulta e outra ou em um determinado período de tempo.

Assim como a dimensão relacional, a dimensão da longitudinalidade foi encontrada quando os usuários referiam-se também aos antigos médicos especialistas:

O doutor é meu médico há 7 anos, 7 ou 8 anos. Aqui, é como se ele fosse da nossa família. Mandaram médicos para cá que não têm o mesmo comprometimento [...]. (G2-Int4).

Nossa, aqui eu já fui várias fases da minha vida, porque foi assim, criança, né? Aí minha mãe trazia, depois mocinha, depois me casei e trago as minhas filhas, e pra mim também, né?” (G1-Int1).

Isso pode ser explicado pela recém-chegada dos médicos da ESF nos CMS e sua rotatividade, o que impede a construção do vínculo e faz com que os usuários expressem essa dimensão referindo-se aos médicos antigos. Para essa dimensão foram consideradas ainda duas subdimensōes: "vínculo" e "cuidados médicos".

Eles já estavam com a gente há muitos anos e conhece [sic] toda a comunidade. Ela fez todo o meu tratamento pré-natal, certo; minha filha nasceu. Depois, passei o acompanhamento dela todo aqui no posto. A minha filha não tem nenhum dente cariado. Tratei da dentição desde bebê aqui no posto, então esses médicos são realmente da família (G2-Int3) 
No contexto brasileiro, a Política Nacional de Atenção Básica (2011), explicita o vínculo como um dos princípios desse nível de atenção e o define como o estabelecimento de relações de confiança e afetividade entre o usuário e o profissional, garantindo a corresponsabilização pela saúde, construída ao longo do tempo, além de ter um potencial terapêutico.

Em todo caso, o vínculo pressupõe, em primeiro plano, uma boa relação profissional-paciente, envolvendo questóes como poder e dependência entre sujeitos com diferentes níveis de informação (CUNHA; GIOVANELLA, 2011).

[...] de 6 em 6 meses eu me consulto aqui porque eu sou hipertenso. Então quando eu chegava aqui, ele falava 'como é que você tá?' 'Eu estou bem, doutor, na medida do possível’ [...]. (G2-Int4)

Essa menção demonstra alguns benefícios que a longitudinalidade pode trazer ao usuário. $\mathrm{O}$ acompanhamento ao longo do tempo e a atenção dispensada à pessoa, e não somente ao manejo dos problemas, oferecem ao usuário um cuidado mais adequado, um reconhecimento melhor dos seus problemas, proporcionando até a menor utilização dos serviços de saúde, uma vez que há um acompanhamento regular.

Já a dimensão "coordenação do cuidado" foi menos referida. Segundo Starfield (2002), a coordenação é o quarto componente da atenção, essencial para os outros aspectos.

Para essa dimensão, encontramos a subdimensão "referência/continuidade". Decidiu-se agrupá-las devido à estreita relação que têm, pois os usuários, quando encaminhados para serviços especializados, dificilmente retornam com informações para o profissional responsável pelo encaminhamento. Isso fragmenta a continuidade da atenção, já que esta necessita que as informaçôes sejam transmitidas. A continuidade é atingida quando há canais de comunicação que permitam a transmissão de informaçóes importantes a respeito dos pacientes por meio de prontuários médicos contendo informaçôes precisas e completas a respeito do paciente.

Além da fragmentação da informação, existe a dificuldade de conseguir um atendimento nos outros níveis de assistência, o que também contribui para interrupção da continuidade da atenção.

Então justamente a Policlínica só atende com encaminhamento, agora. Antigamente, você ia pra porta de entrada e lá ainda era mais fácil de você ser atendido do que aqui na Clínica da Família. (G2-Int1) 
A questão da referência para serviços secundários e terciários, com destaque para algumas especialidades, ainda se configura como um problema. Outra dificuldade seria o tempo de espera que os usuários relataram para conseguir atendimento nos outros níveis de assistência.

Marcou para 6 meses [...]. (G2-Int3)

(... ) mas o problema é que marcaram, mas não sabem para quando. (G2-Int1)

Destaca-se que alguns usuários já reconhecem a ESF como porta de entrada para a atenção básica:

Eu falava muito com o presidente da associação daqui de ele dar um jeito de valorizar mais a comunidade com mais um postinho aqui, que a maioria da gente é daqui. Você vai para outro hospital, mas você passa primeiro por aqui. (G1-Int1)

Há um grande desafio para a construção de políticas referentes à coordenação do cuidado, pois é necessário mais esclarecimento sobre os processos de encaminhamentos, com a definição de motivos e casos em que haja necessidade do paciente ser cuidado por outro profissional que não o da atenção básica. Além disso, é primordial desenvolver a cultura da contrarreferência, para que não ocorra a fragmentação das informações referentes aos usuários.

A estruturação do Núcleo de Apoio à Saúde da Família (NASF), constituído por profissionais de diferentes áreas de conhecimento, poderá minimizar este problema. A corresponsabilização entre a equipe do NASF e as equipes de saúde da família prevê uma nova lógica para a prática do encaminhamento com base nos processos de referência e contrarreferência, ampliando para um processo de discussão conjunta de casos e de acompanhamento longitudinal de responsabilidade das equipes de atenção básica, atuando no fortalecimento de seus princípios e no papel de coordenação do cuidado nas redes de atenção à saúde (BRASIL, 2011).

\section{Considerações finais}

O conceito de satisfação, em especial dos usuários, está intimamente relacionado à qualidade do serviço ofertado à população. Assim, com o intuito de aperfeiçoar os serviços de saúde, é importante investigarmos a satisfação daqueles que usufruem de tais serviços.

A dimensão mais relevante no estudo foi o acesso, revelando a dificuldade de nossa população nesse atributo. No contexto brasileiro, um dos desafios da ESF 
é a ampliação do acesso, descaracterizando o antigo modelo de atenção presente em nossa sociedade. Destaca-se também a dimensão relacional, que exerce grande peso nos estudos encontrados na literatura, sendo um aspecto bastante valorizado pelos nossos usuários.

Os estudos validados e padronizados para avaliar tal conceito são escassos em nosso país. São necessárias mais pesquisas nesse campo no sentido de produzir instrumentos que incorporem as dimensōes relacionadas à satisfação, respeitando a cultura e a especificidade de cada país. Ao utilizamos instrumentos produzidos em outros países, as dimensões da satisfação provavelmente também serão diferentes, já que tratamos de populações com padrões socioeconômicos, culturais e serviços de saúde totalmente distintos.

Explorar o que o usuário pensa e necessita é essencial para que os estudos de satisfação sejam mais proveitosos e sejam, portanto, incorporados à rotina dos serviços, não servindo somente como pontos isolados de reflexão. A incorporação rotineira da avaliação da satisfação pela perspectiva dos usuários deve propiciar sua institucionalização pelos serviços, resultando na melhoria da qualidade dos cuidados e dos serviços prestados.

É fundamental que, antes de avaliarmos a satisfação dos usuários, conheçamos o perfil dessa população, suas necessidades, experiências e expectativas. A estratégia dos grupos focais para identificar as dimensões da satisfação é o passo inicial para o desenvolvimento de qualquer instrumento.

Um instrumento construído através das experiências dos usuários contribui positivamente na avaliação pela perspectiva destes, além de ser útil na mudança de paradigma de como a avaliação da satisfação tem sido conduzida até hoje, deixando, portanto, de se limitar ao uso de questionários "importados" ou "préfabricados".

Por fim, avaliar a satisfação através de instrumentos como este é essencial na medida em que reconhecem os usuários como cidadãos autônomos e protagonistas do seu próprio cuidado. Tornam-se uma ferramenta útil para realizar intervenção, redirecionamento e formulação nos serviços de saúde e nas políticas públicas, propiciando que a qualidade do serviço seja centrada no usuário, e fortalecendo, assim, preceitos chaves estabelecidos pela ESF. ${ }^{1}$ 


\section{Referências}

AHARONY, L.; STRASSER, S. Patient satisfaction: what we know about and what we still need to explore. Medical Care Review, v. 50, n. 1, p. 49-79, 1993.

ANDRADE, A. et al. Programa Qualidade do Ministério da Segurança Social e do Trabalho: um Modelo Integrado de Aplicação da CAF. Lisboa: Secretaria-Geral do Ministério da Segurança Social e do Trabalho, 2004. 188p

COTTA, R.M.M. et al. A satisfação dos usuários do Programa Saúde da Família: avaliando o cuidado em saúde. Scientia Medica. Porto Alegre, v. 15, n. 4, p. 227-234, 2005.

BARATA, R.B. Condições de Saúde da População Brasileira. In: GIOVANELLA, L. et al. (Org.). Políticas e Sistema de Saúde no Brasil. Rio de Janeiro: Fiocruz. 2008. p. 173-175.

BARDIN, L.. Análise de conteúdo. Lisboa: Edições 70, 1979.

BARON-EPEL, O.; DUSHENAT, M.; FRIEDMAN, N. Evaluation of the consumer model: relationship between patients' expectations, perceptions and satisfaction with care. International Journal Quality in Health Care,v. 13, n. 4, p. 317-323, 2001.

BRANDÃO, A.L.R.B.S. Avaliação da atenção básica pela perspectiva dos usuários: adaptação do instrumento Europep para grandes centros urbanos brasileiros - uma aplicação na comunidade de Manguinhos. 2011. 227p. Dissertação (Mestrado em Saúde Pública) Escola Nacional De Saúde Pública, Rio de Janeiro, 2011.

BRASIL. Ministério da Saúde. Política Nacional de Atenção Básica. Brasília: Ministério da Saúde, 2011.

CAMPBELL, S.M.; ROLAND, M.O.; BUETOW, S.A. Defining quality of care. Social Science \& Medicine. Manchester, v. 50, n. 11, p. 1611-1625, 2000.

COTTA, R.M.M.A. Satisfação dos usuários do Programa de Saúde da Família: avaliando o cuidado em saúde. Scientia Medica, Porto Alegre, v. 15, n. 4, p. 227-234, 2005.

CUNHA, E.M.; GIOVANELLA, L. Longitudinalidade/continuidade do cuidado: identificando dimensões e variáveis para a avaliação da Atenção Primária no contexto do sistema público de saúde brasileiro. Ciênc. Saúde Coletiva, Rio de Janeiro, v.6, supl. 1, p. 1029-1042, 2011.

DAWSON, S.; MANDERSON, L.; TALLO, V. A manual for the use offocus groups. Boston: International Nutrition Foundation for Developing Countries, 1993. 96p.

ESPERIDIÃO, M.A.; TRAD, L.A.B. Avaliação de satisfação de usuários: considerações teórico-conceituais. Cad. Saúde Pública, Rio de Janeiro, v. 22, n. 6, p. 1267-1276, 2006.

GOMES, E.S.; BARBOSA, E.F. A técnica de grupos focais para obtenção de dados qualitativos. Instituto de Pesquisa e Inovações Educacionais. (Publicação interna). Fev./1999. 
GULDVOG, B. Can patient satisfaction improve health among patients with angina pectoris? International Journal Quality in Health Care, v. 11, n. 3, p. 233-240, 1999.

HALAL, I.S. et al .Avaliação da qualidade de assistência primária à saúde em localidade urbana da região sul do Brasil. Rev. Saúde Pública, São Paulo, v. 28, n. 2, p. 131-136, 1994. JORGE, M.S.B. et al. Avaliação da qualidade do PSF no Ceará: a satisfação dos usuários. Revista Baiana Saúde Pública, Salvador, v. 31, n. 2, p. 256-206, 2007.

KOTAKA, F.; PACHECO, M.L.R.; HIGAKI, Y. Avaliação pelos usuários dos hospitais participantes do programa de qualidade hospitalar no Estado de São Paulo, Brasil. R. Saúde Pública, São Paulo, v. 31, n. 2, p.171-177, 1997.

KRUEGER, R.; CASEY, M.A. Focus Groups: A Practical Guide for Applied Research. Londres: Sage Publications, 1996. 320p.

LINDER-PELZ, S. Towards a Theory of Patient Satisfaction. Social Science and Medicine. Israel, v. 16, n. 5, p. 577-582, 1982.

MISHIMA, S.M. et al. A assistência na saúde da família sob a perspectiva dos usuários. Revista Latino Americana de Enfermagem, São Paulo, v.18, n. 3, p.148-55, 2010.

PARASURAMAN, A.; ZEITHAML, V.A.; BERRY, L.L. Servqual: A multiple-item for scale for measuring consumer perceptions of service quality. Journal of Retailing. Cambridge, v. 64, n. 1, p. 12-40, 1988.

PRÉVOST, A.; FAFARD, A.; NADEAU, M.A. La mesure de la satisfaction des usagers dans le domaine de la santé et des services sociaux: l'experience de la Régie Régionale Chadière Appalaches.The Canadian Journal of Program Evaluation, v. 13, n. 1, p. 1-23, 1998.

QUEIROZ, M.S. Estratégias de consumo em saúde entre famílias trabalhadoras. Cad. Saúde Públ., Rio de Janeiro, v. 9, n. 3, p. 272-282, 1993.

RIGGE, M. What are patients looking for? In: VAN ZWANENBERG, T.; HARRISON, J. (Orgs.) Clinical Governance in Primary Care. Oxford: Radcliffe Medical Press Ltd, 2009. P.55-62.

RONZANI, T.M.; SILVA, C.M. O Programa Saúde da Família segundo profissionais de saúde, gestores e usuários. Ciênc. saúde coletiva, Rio de Janeiro, v. 13, n. 1, p. 23-34, 2008.

SANTOS, O. et al. Missão para os Cuidados de Saúde Primários. Lisboa: Ministério da Saúde, 2007. 255p.

SECLEN-PALACIN, J.; DARRAS, C. Satisfacción de usuarios de los servicios de salud: factores sociodemográficos y de accesibilidad asociados. Anales de la Facultad de Medicina, Lima, v. 66, n. 2, p. 127-141, 2005.

SILVA, J. et al. Avaliação do nível de satisfação dos usuários do Programa de Saúde da Família no Bairro Furtado Menezes - Juiz de Fora/MG. In: ENCONTRO NACIONAL DE ESTUDOS POPULACIONAIS-ABEP, XIV. Caxambu, 2004. p. 1-13. 
SITZIA, J., WOOD, N. Patient satisfaction: A review of issues and concepts. Social Science and Medicine, v. 45, n. 12, p. 1829-1843,1997.

STARFIELD, B. Atenção Primaria: equilíbrio entre necessidades de saúde, serviços e tecnologia. Brasília: UNESCO, 2002. 725p.

SZWARCWALD, C.L. et al. Desigualdades socioeconômicas em saúde no Brasil: resultados da Pesquisa Mundial de Saúde, 2003. Revista Brasileira de Saúde Materno-Infantil, Recife, v. 5, sup. 1, p. 1-22, 2005.

TEIXEIRA, S.A. Avaliação dos usuários sobre o Programa de Saúde da Família em Vitória da Conquista-Bahia. In: SECLEN, J. (Org.). Experiências e desafios da atenção básica e saúde familiar: caso Brasil. Brasília: OPAS, 2004. p. 77-101.

TRAD, L.A.B. et al. Estudo etnográfico da satisfação do usuário do Programa de Saúde da Família (PSF) na Bahia. Ciência \& Saúde Coletiva, Rio de Janeiro, v. 7, n. 3, p. 581-589, 2002.

VAITSMAN, J.; ANDRADE, G.R.B. Satisfação e responsividade: formas de medir a qualidade e a humanização da assistência à saúde. Ciência \& Saúde Coletiva, Rio de Janeiro, v. 10, n. 3, p.599-613, 2005.

VAN STRALEN, C.J. et al. Percepção dos usuários e profissionais de saúde sobre a atenção básica: comparação entre unidades com e sem saúde da família na Região Centro-Oeste do Brasil. Caderno de Saúde Pública. Rio de Janeiro, v. 24, supl 1, p. 148-158, 2008.

WILLIAMS, B. Patient satisfaction: a valid concept? Social Science Medicine, Wales, v. 38, n. 4, p.509-516, 1994.

\section{Nota}

${ }^{1}$ V.D. Moraes participou da concepção, delineamento, análise, interpretação dos dados, redação do artigo e aprovação final da versão a ser publicada. C.E.A. Campos participou da concepção, redação do artigo, revisão crítica relevante do conteúdo intelectual e aprovação final da versão a ser publicada. A. L. Brandão participou da concepção, redação do artigo, revisão crítica relevante do conteúdo intelectual e aprovação final da versão a ser publicada. 


\section{Abstract}

\section{Study on evaluation of dimensions of the Family Health Strategy in the user's perspective}

The satisfaction of users is one of the basic components of the quality of any health service. The incorporation of this aspect in evaluative studies of units and primary health care teams has been increasing lately. In Brazil, this process is still embrionary, few are available and have appropriate and valid instruments. This paper aimed to investigate the dimensions which are most valued by the users. Two focal groups in two primary health care units with distinct contexts were conducted in order to expand the possibilities to the responses. The researcher held the analysis of the content and the categorization of speeches into dimensions and sub-dimensions of satisfaction. A total of 17 dimensions was achieved, which the most cited were: 1 ) access/availability to health service; 2) organization of the work processes; 3 ) relationship with health professionals; 4) care longitudinality and establishment of bond between professionals and users; and 5) care coordination. The knowledge of the dimensions of satisfaction which are most valued by users is vital to the construction of instruments that will be more suitable to context of the Family Health Strategy in our country.

> Key words: health evaluation; user's satisfaction; Primary Health Care. 